\title{
ARTIGO ORIGINAL \\ Identificação de risco da pessoa idosa em uma instituição de longa permanência para idosos
}

\author{
Waldere Fabri Pereira Ribeiro, D.Sc. ${ }^{*}$, Sara Maria Campos ${ }^{* *}$, Mirilene de Moraes Tunice ${ }^{\star * *}$ \\ ${ }^{*}$ Enfermeira, docente da Escola de Enfermagem Wenceslau Braz, Itajubá/MG, ${ }^{* * E n f e r m e i r a ~ d o ~}$ \\ Lar da Providência, Especialista em Urgência e Emergência e Terapia Intensiva, enfermeira do \\ Lar da Providência de Itajubá/MG, ${ }^{* * *}$ Enfermeira, Especialista em Urgência e Emergência e \\ Terapia Intensiva, enfermeira do Santos Dumont Hospital, São José dos Campos/SP
}

Recebido em 20 de junho de 2015; aceito em 12 de fevereiro de 2016.

Endereço de correspondência: Waldere Fabri Pereira Ribeiro, Rua Olavo Bilac, 31, 37500184 Itajubá MG, E-mail: walfabri@gmail.com

\section{Resumo}

Este artigo propôs-se a realizar um estudo descritivo, documental e retrospectivo com a finalidade de caracterizar as pessoas idosas quanto ao sexo e idade em uma Instituição de Longa Permanência para Idosos, além de identificar o risco dessas pessoas. Aplicando uma abordagem quantitativa, foram utilizadas 57 fichas de cadastro dos idosos internados durante 0 mês de janeiro de 2015. A coleta de dados foi realizada em fevereiro de 2015 e apresentou os seguintes resultados: $54,38 \%$ dos idosos eram do sexo masculino e $45,62 \%$ do sexo feminino. Desses, 26,31\% tinham de 75 a 79 anos. Quanto à classificação de risco, 40,35\% possuíam mais de 80 anos e $59,65 \%$ mais de 60 anos. Sobre os itens que classificam o idoso como frágil, os resultados foram: $71,92 \%$ eram portadores de incontinência urinária; $64,91 \%$ incontinência fecal e 57,89\% apresentaram dependência para atividades do dia a dia. Quanto às incapacidades dos idosos, $22,80 \%$ possuíam incapacidade em vários itens acima citados. Em relação às doenças, $82,45 \%$ possuíam osteoporose; $70,17 \%$ hipertensão arterial; $54,38 \%$ diabetes mellitus; $56,17 \%$ infecção urinária; $38,59 \%$ algum tipo de doença mental e $28,07 \%$ alterações endocrinológicas. Quanto aos medicamentos, $92,98 \%$ dos idosos faziam uso de hipotensor; $78,94 \%$ de protetor gástrico e $70,17 \%$ de antipsicótico. Portanto, conclui-se que é importante orientar familiares e equipe de enfermagem para uma assistência adequada e direcionada à pessoa idosa, oferecendo qualidade no atendimento e cuidados específicos que previnam acidentes e ofereçam possibilidade de manutenção da independência funcional existente que permita ao idoso a realização das atividades da vida diária, consciente da exposição aos riscos de trauma que ele pode vir a sofrer.

Palavras-chave: idoso, enfermagem, risco, instituição de longa permanência para idosos.

\begin{abstract}
\section{Risk identification in elderly people in a long stay institution for the elderly}

This descriptive, documental and retrospective study aimed at characterizing older people according to their age and sex in a long stay institution for the elderly, in addition to identify these people's risk. Applying a quantitative approach, 57 registration forms of elderly admitted during January 2015 were used. Data collection were carried out in February 2015 with the following result: $54.38 \%$ older men and $45.62 \%$ women. Out of these, $26.31 \%$ were 75 to 79 years old. As regard to risk rate, $40.35 \%$ were over 80 years old and $59.65 \%$ over 60 years old. Concerning items which classify the elderly as fragile, the results were: $71.92 \%$ had urinary incontinence; $64.91 \%$ fecal incontinence and $57.89 \%$ showed dependence in personal activities of daily living. $22.80 \%$ had disabilities on various items mentioned above. In relation to the diseases, $82.45 \%$ had osteoporosis; $70.17 \%$ high blood pressure; $54.38 \%$ diabetes mellitus; $56.17 \%$ urinary infection; $38.59 \%$ any mental disease and $28.07 \%$ endocrine changes. With reference to medicines, $92.98 \%$ related to use hypotensive; $78.94 \%$ gastric protection and 70.17 antipsychotic treatment. We concluded that family and nursing staff should receive orientation related to an appropriate and directed assistance to older people, offering quality on attendance and specific cares to prevent accidents, and the possibility of maintaining functional independence to perform activities of daily living, conscious that they are at risk of trauma.
\end{abstract}

Key-words: older people, nursing, risk, homes for the aged. 


\section{Resumen \\ Identificación de riesgo de mayores en una institución de larga permanencia}

Estudio descriptivo, documental y retrospectivo que tuvo por finalidad caracterizar las personas mayores cuanto a la edad y sexo en una Institución de Larga Estancia para Mayores, además de identificar el riesgo de esas personas. Aplicando un enfoque cuantitativo, fueron utilizados 57 registros de ancianos internados durante el mes de enero del 2015. Los datos fueron recolectados en febrero del 2015 y presentó los siguientes resultados: $54,38 \%$ de los ancianos eran del sexo masculino y $45,68 \%$ del sexo femenino. De los cuales $26,31 \%$ tenían entre 75 y 79 años. A respecto de la clasificación de riesgo, 40,35\% poseía más de 80 años y $59,65 \%$ más de 60 . Sobre los puntos que clasifican a los ancianos como personas frágiles, los resultados fueron: $71,92 \%$ eran portadores de incontinencia urinaria, $64,91 \%$ incontinencia fecal y 57,89\% necesitan ayuda para realizar actividades del día a día. A respecto de la incapacidad de los ancianos, 22,80\% poseían incapacidad en varios puntos citados anteriormente. En relación a las enfermedades, $82,45 \%$ poseían osteoporosis; $70,17 \%$ hipertensión arterial; $54,38 \%$ diabetes mellitus; $56,17 \%$ infección urinaria; $38,59 \%$ algún tipo de enfermedad mental y $28,07 \%$ alteraciones endocrinas. A respecto de los medicamentos, $92,98 \%$ de los ancianos hacían uso del hipotensor, 78,94\% de protector gástrico y 70,17\% de antipsicótico. Por lo tanto, se concluye que esimportante orientar a familiares y equipos de enfermería para una asistencia adecuada y direccionada a la persona de la tercera edad, ofreciendo calidad en la atención y cuidados específicos para la prevención a accidentes y que ofrezcan la posibilidad de independencia funcional que permita al anciano la realización de actividades de la vida diaria, consciente de la exposición al riesgo de traumas que él podrá sufrir.

Palabras-clave: anciano, enfermería, riesgo, hogares para ancianos.

Introdução

Preliminarmente é importante ressaltar que, no Brasil, é considerada idosa a pessoa com 60 anos ou mais, enquanto que nos países desenvolvidos, idosos são aqueles que têm 65 anos ou mais [1]. O termo envelhecimento é empregado para descrever as mudanças morfofuncionais que ocorrem ao longo da vida após a maturação sexual e que, progressivamente, comprometem a capacidade de resposta dos indivíduos ao estresse ambiental e a manutenção da homeostasia [2].

Atualmente, o envelhecimento é um grande desafio para o mundo atual, tanto dos países ricos quanto para os países pobres. Segundo a Organização das Nações Unidas (ONU) a população considerada idosa, isto é, com mais de 60 anos, já corresponde a mais de $12 \%$ da população mundial e até a metade deste século, este percentual tende a subir atingindo a marca dos 20\%. Em 2050, prevê-se que o número de pessoas com 100 anos e em pleno vigor físico e mental será surpreendente [3].

Segundo a Organização Mundial de Saúde (OMS), em 2025, o grupo de idosos no Brasil deverá ter aumentado 15 vezes, enquanto a estimativa para a população total é de aumentar apenas cinco vezes com relação à atual. Ainda no mesmo ano, o país contará com um contingente de aproximadamente 32 milhões de pessoas na chamada terceira idade, e a proporção de pessoas com mais de 80 anos continuará em ascensão [4,5].

Um fator característico da população que envelhece no Brasil é a pobreza, já que aposentadorias e pensões são suas únicas fontes de rendimento e seus baixos valores são insuficientes para o provimento de todas as necessidades do idoso que reside numa comunidade. A situação agrava-se quando o idoso é acometido de uma ou mais doenças crônicas que provocam o comprometimento de sua independência. Além disso, é nesta fase da vida - acima dos 60 anos - que as pessoas mais utilizam serviços de investigação diagnóstica e consomem mais serviços hospitalares e ambulatoriais [6,7].

Antes do adoecimento orgânico, a pessoa idosa apresenta alguns sinais de risco. Deste modo, é função do profissional de saúde identificá-los para que as ações de enfermagem possam ser adotadas de maneira precoce, contribuindo, assim, não apenas para a melhoria da qualidade de vida individual, mas também para uma saúde pública mais consciente e eficaz.

Identificar os fatores de risco e os problemas de saúde que levam um idoso à síndrome de fragilidade - síndrome multidimensional constituída por fatores biológicos, psicológicos e 
sociais em uma interação complexa no curso de vida individual - é de fundamental importância, uma vez que a partir deste conhecimento pode-se traçar um plano de cuidado para o idoso. Deste modo, são elaboradas estratégias eficazes para a promoção da saúde e bem-estar desta faixa etária por meio de ações educativas, grupos de convivência, tratamento adequado das doenças e encaminhamento para centros de referência quando se fizer necessário [8].

Diante de tais fatos e cientes de que a saúde da pessoa idosa e o envelhecimento são preocupações relevantes do Ministério da Saúde (MS), além de ser uma das áreas estratégicas do Departamento de Ações Programáticas e Estratégicas (DAPES) do MS, é possível perceber que classificar os idosos quanto à sua fragilidade é uma forma de conhecer a realidade com a qual se trabalha. Há, diante disso, a possibilidade de propor estratégias de intervenção que terão melhor impacto sobre a qualidade de saúde destes idosos.

Para a Agência Nacional de Vigilância Sanitária (ANVISA), as Instituições de Longa Permanência para Idosos (ILPIs) são governamentais ou não governamentais, de caráter residencial, destinadas a domicílio coletivo de pessoas com idade igual ou superior a 60 anos, com ou sem suporte familiar, em condição de liberdade, dignidade e cidadania [9].

Por este motivo, caracterizar as pessoas idosas quanto ao sexo e idade em uma ILPI de Itajubá/MG e identificar o risco de tais pessoas, justifica a realização deste estudo. Afinal, ao utilizar o instrumento para cadastro e identificação de risco da pessoa idosa proposta pela Coordenação Estadual de Atenção ao Idoso da Secretaria de Saúde de Minas Gerais (SES/MG), é possível avaliar, de maneira padronizada, o grau de fragilidade dos idosos assistidos pela equipe de enfermagem da ILPI em questão [10].

\section{Material e métodos}

Este estudo caracteriza-se como quantitativo, descritivo e retrospectivo, fundamentado na análise de um documento denominado Ficha de Cadastro e Identificação de Risco da Pessoa Idosa que compõe o prontuário dos pacientes internos no Lar da Providência de Itajubá, Minas Gerais. Tal documento encontrava-se arquivado, completo, organizado e constava de três partes, sendo elas: dados de identificação do idoso; classificação de risco e outras doenças.

Para a realização da pesquisa, foram utilizadas 57 fichas de cadastro e identificação de risco da pessoa idosa que faziam parte do prontuário dos internos no mês de janeiro de 2015 na referida instituição. A partir desse documento, a coleta de dados foi realizada durante o mês de fevereiro de 2015, quando foram agendados antecipadamente os dias e horários em que as pesquisadoras realizaram a coleta dos dados. Os dados coletados foram tabulados e analisados através do programa Microsoft Office Excel.

Foi solicitada uma autorização para a coleta de dados e desenvolvimento da pesquisa para a Diretora da instituição. Após a autorização, o projeto foi encaminhado ao Comitê de Ética e Pesquisa, sendo aprovado conforme Parecer Consubstanciado número 754.059. A pesquisa seguiu os preceitos da Resolução 466/12 do Ministério da Saúde no que diz respeito à autonomia, não-maleficência, beneficência, justiça e asseguração aos direitos e deveres que dizem respeito à comunidade, aos sujeitos da pesquisa e ao Estado [11].

\section{Resultados e discussão}

A apresentação dos resultados e discussão foi dividida em três partes apresentadas em tabelas. A tabela I demonstra as características pessoais dos idosos, a tabela II revela a classificação de risco e as tabelas III e IV indicam as doenças apresentadas pelos idosos.

As características referentes aos aspectos pessoais dos idosos em uma ILPI encontram-se na tabela I, a seguir. 
Tabela I - Características pessoais dos idosos em relação ao sexo e idade em uma ILPI. Itajubá/MG $(N=57)$.

\begin{tabular}{lcc}
\hline Sexo & f & $\%$ \\
\hline Masculino & 31 & 54,38 \\
Feminino & 26 & 45,62 \\
Idade & $\mathbf{f}$ & $\%$ \\
\hline $60-64$ & 6 & 10,52 \\
$65-69$ & 8 & 14,03 \\
$70-74$ & 5 & 8,78 \\
$75-79$ & 15 & 26,31 \\
$80-84$ & 14 & 24,56 \\
$85-90$ & 4 & 7,02 \\
$>90$ & 5 & 8,78 \\
\hline
\end{tabular}

Fonte: Elaborada pelos autores; Nota: Informações extraídas do Instrumento de Pesquisa.

A tabela acima faz referência a uma amostra de 57 fichas de cadastro e identificação de risco da pessoa idosa. Os idosos participantes do estudo foram 31 do sexo masculino e 26 do sexo feminino.

Quanto à idade observou-se que a prevalência é de 75 a 79 anos, totalizando 15 $(26,31 \%)$ idosos. Porém, é notável que a faixa de idade entre 80 a 84 anos possui um número significativo de $14(24,56 \%)$ idosos da ILPI. Desta forma, é possível concluir que mais da metade dos idosos possuem idade acima de 75 anos.

A Classificação de Risco dos idosos em uma ILPI encontram-se na tabela II.

Tabela II - Classificação de Risco dos idosos em uma ILPI. Itajubá/MG ( $N=57)$.

\begin{tabular}{lcc}
\hline \multicolumn{1}{c}{ Classificaçăo } & $\mathbf{f}$ & $\%$ \\
\hline icima de 80 anos & 23 & 40,35 \\
j0 an os ou mais & 34 & 59,65 \\
Riscos & & \\
Incontinência urinária & 41 & 71,92 \\
Incontinênciafecal & 37 & 64,91 \\
Usa mais de cinco medicamentos continuamente & 25 & 43,85 \\
Esquecimento progressivo que impede atividades nomais & 28 & 49,12 \\
Sofreu queda nos últimos seis meses & 14 & 24,56 \\
Foi internado nos últimos seis meses & 22 & 38,59 \\
Está acamado & 20 & 35,08 \\
Tem dependência para atividades do dia-a-dia & 33 & 57,89 \\
Econ siderado idoso frágil & $\mathbf{5 7}$ & $\mathbf{1 0 0}$ \\
\hline
\end{tabular}

Fonte: Elaborada pelos autores; Nota: Informações extraídas do Instrumento de Pesquisa.

Quanto à classificação de risco da pessoa idosa, foi possível observar que todos os idosos são considerados frágeis, visto que 23 (40,35\%) possuem mais de 80 anos e os outros idosos $34(59,65 \%)$ apresentam mais de 60 anos e dispõem de um ou mais dos itens descritos acima, sendo, portanto, classificados como "idosos frágeis".

Destes itens, é necessário observar que $41(71,92 \%)$ idosos possuem incontinência urinária, $37(64,91 \%)$ incontinência fecal e $33(57,89 \%)$ possuem dependência para atividades do dia-adia.

A queda é um dos principais eventos que acomete a pessoa idosa e pode indicar o início de fragilidade ou mesmo apontar doenças agudas que levam a custos sociais, econômicos e psicológicos que não passam despercebidos. Dessa maneira, há significativo aumento da dependência que já havia e muitas vezes à institucionalização. Ainda, segundo o Ministério da Saúde, os riscos dependem da frequência de exposição do idoso ao ambiente inseguro e do estado funcional do idoso [4].

A incontinência urinária e fecal aparece em cerca de $30 \%$ das pessoas idosas institucionalizadas ou não. Muitas das causas são reversíveis visto que podem originar-se em períodos de delírio, restrição de mobilidade, retenção urinária, infecção se ocasionada por efeito de medicamentos e devem ser investigadas. Em muitos casos, tais incontinências estão relacionadas às repercussões emocionais e sociais [10]. Além disso, as incapacidades funcionais para as atividades básicas de vida diária referem-se à deambulação, alimentação, higiene, mudanças, vestuário e continência. 
A incapacidade de autocuidado dificulta a tomada de decisões sobre o futuro, resultando na renúncia de sua própria responsabilidade. Alguns idosos delegam tal responsabilidade de maneira absoluta aos profissionais de saúde ou para os seus familiares [12].

As mudanças e perdas fazem parte do envelhecimento da pessoa humana e modificações corporais e mentais ocorrem ao longo do envelhecimento, um processo caracterizado por perdas [13].

O demonstrativo de doenças e outras doenças que não estão listadas na ficha de cadastro e identificação de risco da pessoa idosa em uma ILPI, encontram-se nas tabelas III e IV.

Tabela III - Doenças apresentadas pelos idosos em uma ILPI. Itajubá/MG. ( $N=57)$.

\begin{tabular}{lcc}
\hline Doen ças & $\mathrm{f}$ & $\%$ \\
\hline Osteoporose & 47 & 82,45 \\
Hipertensão Arterial Sistêmica & 40 & 70,17 \\
Diabetes & 31 & 54,38 \\
Doen ça de Alzheimer & 16 & 28,07 \\
Acidente Vascular Cerebral & 15 & 26,31 \\
Doen ça Pulmonar Obstrutiva Crônica & 9 & 15,78 \\
Pneumonia & 7 & 12,28 \\
Alcoolismo & 7 & 12,28 \\
Doen ça de Parkin son & 4 & 7,01 \\
Fratu ras & 4 & 7,01 \\
\hline
\end{tabular}

Fonte: Elaborada pelos autores; Nota: Informações extraídas do Instrumento de Pesquisa.

Sobre as doenças listadas na ficha de cadastro e identificação de risco da pessoa idosa, a tabela acima demonstra que $47(82,45 \%)$ idosos possuem osteoporoses, $40(70,17 \%)$ idosos possuem hipertensão arterial sistêmica e 31 (54,38\%) idosos possuem diabetes mellitus.

Sabe-se que não existem doenças próprias da pessoa idosa. Porém, há situações em que apelidos pejorativos são utilizados para caracterizar as pessoas dessa idade, tal como "velho gagá", mais empregado para denominar a pessoa idosa portadora de demência.

Uma evidente deterioração da qualidade de vida da pessoa com osteoporose pode ser detectada, não excluindo a possibilidade de estar associada à mortalidade, especialmente por complicações como infecções e problemas cardiovasculares. Desta forma, trata-se de um crescente problema de saúde pública [14].

A deterioração de órgãos e de sistemas ocorre de maneira progressiva no processo de envelhecimento, além de ser acompanhado de perdas no âmbito social como a aposentadoria, o isolamento e também perdas econômicas e familiares, ou seja, falecimentos e doenças de pessoas próximas [12].

A peculiaridade das doenças geriátricas é justificada pela deterioração que é acarretada sobre aspecto funcional, mental e social do idoso [12].

Tabela IV - Outras doenças confirmadas nos idosos em uma ILPI. Itajubá/MG ( $N=57)$.

\begin{tabular}{lcc}
\hline Outras doenças & f & $\%$ \\
\hline Cardíacas & 9 & 15,78 \\
Mentais & 22 & 38,59 \\
Musculoesqueléticas & 8 & 14,03 \\
Neoplásicas & 3 & 5,26 \\
Órgão dos sentidos & 5 & 8,78 \\
Circulatórias & 13 & 22,80 \\
Infecção Urinária & 32 & 56,17 \\
Endocrinológicas & 16 & 28,07 \\
Musculares & 4 & 7,01 \\
\hline
\end{tabular}

Fonte: Elaborada pelos autores; Nota: Informações extraídas do Instrumento de Pesquisa.

A tabela acima informa outras doenças que os idosos do estudo possuem: $32(56,17 \%)$ idosos possuem infecção urinária, $22(38,59 \%)$ têm algum tipo de doença mental e $16(28,07 \%)$ idosos são portadores de alterações endocrinológicas.

A tabela também aponta que os idosos apresentam patologias com uma frequência muito elevada (algumas estatísticas chegam até $80 \%$ ). As doenças mais comuns em geriatria são as cardiovasculares, respiratórias, digestivas, urinárias, metabólicas e endócrinas, 
psiquiátricas dentre outras [12,15]. Frequentemente coexiste mais de uma doença, o que pode ser denominado de pluripatologia.

O resultado sobre as doenças que mais acometem os idosos na ILPI, local de realização da pesquisa, se assemelha ao de outro estudo realizado em uma ILPI no extremo sul do Rio Grande do Sul, onde foram encontradas três patologias em grande escala, apresentadas, neste artigo, em ordem decrescente: a hipertensão arterial sistêmica, o diabetes mellitus e a depressão [16].

Em relação às características pessoais dos 57 internos na referida ILPI, 54,38\% eram do sexo masculino e $45,62 \%$ do sexo feminino, estando $26,31 \%$ na faixa etária de 75 a 79 anos de idade.

Em relação à classificação de risco da pessoa idosa, 40,35\% possuíam mais de 80 anos e $59,65 \%$ possuíam mais de 60 anos. Sobre um ou mais itens que classificam o idoso como frágil, resumem-se em: $71,92 \%$ possuem incontinência urinária; $64,91 \%$ incontinência fecal e $57,89 \%$ apresentam dependência para atividades do dia a dia.

Sobre os itens que se referem às doenças listadas na ficha de cadastro e identificação de risco da pessoa idosa: $82,45 \%$ possuíam osteoporose; $70,17 \%$ possuíam hipertensão arterial sistêmica e $54,38 \%$ possuíam diabetes mellitus. Os números em relação às outras doenças indicam que $56,17 \%$ possuíam infecção urinária; $38,59 \%$ possuíam algum tipo de doença mental e $28,07 \%$ alterações endocrinológicas.

$O$ processo de envelhecimento, suas consequências naturais e as alterações biomorfológicas são preocupações da humanidade desde o início da civilização. Naturalmente, os seres vivos são regidos por um determinismo biológico: todos nascem, crescem, amadurecem, envelhecem, declinam e morrem [17].

Estudos concluem que um desempenho cognitivo não favorável nos idosos parece estar relacionado com a institucionalização [18]. Portanto, a desconsideração do idoso institucionalizado como morador da área de abrangência dos serviços de atenção básica à saúde pode ser considerada o pano de fundo na comunicação entre as ILPIs e o Sistema Único de Saúde. Geralmente, a responsabilidade pelo cuidado com o idoso é repassada a estas instituições, havendo, como ressonância, a exclusão do idoso das ações programáticas em saúde. Por outro lado, há desconhecimento, por parte das ILPIs, sobre os serviços de atenção à saúde direcionada ao idoso pelo Sistema Único de Saúde [19].

Porém, não é o que ocorreu na ILPIs em questão, visto que no prontuário dos idosos internados a ficha de cadastro e identificação de risco da pessoa idosa estava devidamente preenchida. Desta forma, foi possível avaliar, de maneira padronizada, o grau de fragilidade dos idosos assistidos pela equipe de enfermagem da ILPI em questão. A partir deste conhecimento, é possível oferecer um atendimento individualizado de melhor qualidade, com o objetivo de oferecer qualidade de vida, além de prevenir acidentes com os idosos e ensinar práticas de autocuidado. É necessário lembrar que a pessoa idosa requer diferentes níveis de intervenção dos serviços de saúde, e que tais serviços devem ser adequados às diferentes fases da enfermidade e ao grau de incapacidades. Desta forma os cuidadores e os familiares dos idosos podem tornar-se multiplicadores dos seus conhecimentos da área de saúde.

Conhecer sobre a classificação de risco para a pessoa idosa pode dar condições de orientar familiares e equipe de enfermagem para uma assistência adequada e direcionada à pessoa idosa, proporcionando qualidade no atendimento e cuidados específicos que previnam acidentes. Dessa maneira, é possível oferecer possibilidade de manutenção da independência funcional existente que habilite o idoso a realizar atividades da vida diária, consciente de que pode haver uma exposição aos riscos de trauma. Também são necessárias atividades diárias de estimulação cognitiva para os idosos institucionalizados com o objetivo de melhoria do desempenho cognitivo.

Deste cenário emerge a necessidade de maiores estudos considerando a importância de avaliação sobre a saúde da pessoa idosa, pois ela inclui diversos fatores, tais como ambientais, socioeconômicos, culturais e políticos, itens que vão além do fator saúde, apenas. A pessoa idosa não pode ser considerada como sinônimo de doença. Não se fica idoso aos 60 anos. O envelhecimento é um processo natural que se desenvolve ao longo de toda a vida do ser humano, por meio de diversos fatores decorrentes das escolhas e das circunstâncias da vida. 
1. Word Health Organization (WHO). Envelhecimento ativo: uma política de saúde. Brasília: Organização Pan-Americana da Saúde; 2006.

2. Araújo CLO. Condições de saúde e funcionalidade dos idosos do Vale do Paraíba - SP [Tese]. São Paulo: Universidade Federal de São Paulo, Escola de Enfermagem da Universidade Federal de São Paulo; 2012.

3. Camarano AAA. A demografia e o envelhecimento populacional. In: Abreu APB (Org.). Envelhecimento e saúde da pessoa idosa. Rio de Janeiro: EAD/ENSP, 2008. p.111-34.

4. Brasil. Instituto Brasileiro de Geografia e Estatística (IBGE). Indicadores Sociodemográficos e de saúde no Brasil. Sobre a condição de saúde dos idosos: indicadores selecionados. Rio de Janeiro: IBGE; 2009.

5. Carvalho Filho ET, Papaleo Netto M. Geriatria: fundamentos, clínica e terapêutica. São Paulo: Atheneu; 2000.

6. Chaimowicz F. Saúde do idoso. Belo Horizonte: Nescon UFMG/COOPMED; 2013.

7. Oliveira JC. Envelhecimento populacional e doenças crônicas: tendências de um novo mercado de trabalho e de consumo de serviços de saúde na terceira idade. In: Barros Júnior JC (Org). Empreendedorismo, trabalho e qualidade de vida na $2^{a}$ idade. Porto: Legis; 2009.

8. Brasil. Ministério da Saúde. Secretaria de Atenção à saúde. Departamento de Atenção Básica. Envelhecimento e saúde da pessoa idosa [Internet]. Brasília: Ministério da Saúde; 2007.

9. Camarano AA, Kanso S. As instituições de longa permanência para idosos no Brasil. Rev Bras Estud Popul 2010;27(1):232-5.

10. Minas Gerais. Secretaria de Estado de Saúde. Atenção à Saúde do Idoso. 2 ed. Belo Horizonte: SAS/MG; 2007.

11. Brasil. Resolução № 466, de 12 de dezembro de 2012, Pesquisa envolvendo seres humanos [Internet]. 2012. [citado 2013 Set 10]. Disponível em URL: http://conselho.saude.gov.br/resolucoes/2012/Reso466.pdf

12. Ruipérez I, Liontene P. Geriatria. Rio de Janeiro: MacGrawHill; 1998.

13. Costa EFA, Pereira SRM. Meu corpo está mudando o que fazer? In: Pacheco JLM, ed. Tempo: rio que arrebata. Holambra: Setembro; 2005.

14. Castro ML, Eis SR, Marques Neto JF. A prevenção da osteoporose levada a sério: uma necessidade nacional. Arq Bras Endocrinol Metab 2008;52(4):712-3.

15. Florindo S, Caetano D, Pacheco JL, Sé EVG, Lacerda ALT. Factors influencing psychotropic prescription by non-psychiatrist physicians in a nursing home for the elderly in Brazil. São Paulo Med J 2006;124(5):253-6.

16. Ramos CV. Doenças crônicas não transmissíveis que mais acometem os idosos residentes em uma ILPI no extremo sul do Rio Grande do Sul. Periódicos UEM [Internet]. 2013. [citado 2015 Jan 23]. Disponível em URL: http://periodicos.uem.br/ojs/index.php/CiencCuidSaude/article/viewFile/20899/pdf

17. Silva AC. Processo natural do envelhecimento. In: Silva JV. Saúde do Idoso Processo de Envelhecimento sob Múltiplos Aspectos. São Paulo: látria; 2009.

18. Plati MCF, Covre P, Lukasova K, Macedo EC. Depressive symptoms and cognitive performance of the elderly: relationship between institutionalization and activity programs. Rev Bras Psiquiatr 2006;28(2):118-21.

19. Creutzberg M, Gonçalves LHT, Sobottka EA, Ojeda BS. Long-term care institutions for elders and the health system. Rev Latinoam Enferm 2007;15(6):1144-9. 